\title{
ARTICLE
}

Clinical Research

\section{ZRSR2 overexpression is a frequent and early event in castration- resistant prostate cancer development}

\author{
Haiqing $\mathrm{He} \mathbb{C}^{1,2} \cdot$ Jun $\mathrm{Hao}^{1} \cdot$ Xin Dong ${ }^{3} \cdot \mathrm{Yu}$ Wang $\mathbb{1}^{1,3} \cdot \mathrm{Hui}^{\mathrm{Xue}}{ }^{3} \cdot$ Sifeng $\mathrm{Qu}^{4,5} \cdot$ Stephen Yiu Chuen Choi ${ }^{1,3}$.

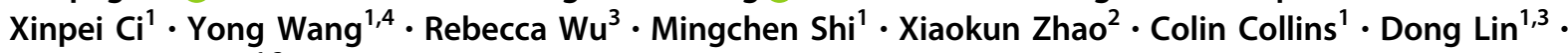 \\ Yuzhuo Wang $\mathbb{1}^{1,3}$
}

Received: 23 August 2020 / Revised: 1 December 2020 / Accepted: 9 January 2021 / Published online: 10 February 2021

(C) The Author(s) 2021. This article is published with open access

\begin{abstract}
Background Androgen deprivation therapy (ADT) remains the leading systemic therapy for locally advanced and metastatic prostate cancers (PCa). While a majority of PCa patients initially respond to ADT, the durability of response is variable and most patients will eventually develop incurable castration-resistant prostate cancer (CRPC). Our research objective is to identify potential early driver genes responsible for CRPC development.

Methods We have developed a unique panel of hormone-naïve PCa (HNPC) patient-derived xenograft (PDX) models at the Living Tumor Laboratory. The PDXs provide a unique platform for driver gene discovery as they allow for the analysis of differentially expressed genes via transcriptomic profiling at various time points after mouse host castration. In the present study, we focused on genes with expression changes shortly after castration but before CRPC has fully developed. These are likely to be potential early drivers of CRPC development. Such genes were further validated for their clinical relevance using data from PCa patient databases. ZRSR2 was identified as a top gene candidate and selected for further functional studies. Results ZRSR2 is significantly upregulated in our PDX models during the early phases of CRPC development after mouse host castration and remains consistently high in fully developed CRPC PDX models. Moreover, high ZRSR2 expression is also observed in clinical CRPC samples. Importantly, elevated ZRSR2 in PCa samples is correlated with poor patient treatment outcomes. ZRSR2 knockdown reduced PCa cell proliferation and delayed cell cycle progression at least partially through inhibition of the Cyclin D1 (CCNDI) pathway.

Conclusion Using our unique HNPC PDX models that develop into CRPC after host castration, we identified ZRSR2 as a potential early driver of CRPC development.
\end{abstract}

Supplementary information The online version contains supplementary material available at https://doi.org/10.1038/s41391021-00322-7.

Yuzhuo Wang

ywang@bccrc.ca

1 Vancouver Prostate Centre, Department of Urologic Sciences, University of British Columbia, Vancouver, BC, Canada

2 Department of Urology, The Second Xiangya Hospital, Central South University, Changsha, China

3 Department of Experimental Therapeutics, BC Cancer Research Centre, Vancouver, BC, Canada

4 Department of Urology, Qilu Hospital, Cheeloo College of Medicine, Shandong University, Jinan, Shandong, China

5 School of Medicine, Cheeloo College of Medicine, Shandong University, Jinan, Shandong, China

\section{Introduction}

Androgen deprivation therapy (ADT) remains the first-line treatment for patients with locally advanced and metastatic prostate cancer (PCa) [1]. While a majority of patients initially respond well to ADT, most will progress to castration-resistant prostate cancer (CRPC). Over the last decade, the discovery that most CRPCs are still dependent on androgen and androgen receptor (AR) signaling led to the development of several new androgen receptor pathway inhibitors (ARPIs) such as abiraterone, enzalutamide and apalutamide [2-4]. While these next-generation ARPIs can effectively reduce symptoms and prolong life, metastatic CRPC (mCRPC) remains incurable as resistance to these ARPIs frequently emerges. Furthermore, although multiple ARPIs are currently in clinical use, most of these therapies focus on late stages of the disease. Knowledge surrounding 
the early stages of CRPC development remain limited. As such, a better understanding of the mechanisms that underlie the early phases of CRPC development is needed to develop novel therapeutic approaches.

It is particularly important, yet extremely challenging, to study the early stages of CRPC development. This is largely due to a difficulty in acquiring adequate clinical samples and a lack of suitable patient-derived xenograft (PDX) models that can represent the biology of patient CRPC progression. At the Living Tumor Laboratory (www.livingtumorlab.com), we have previously established a large panel of PDX models from various clinical stages of PCa. In particular, we have a unique panel of PCa PDXs originating from hormonal-naïve PCa (HNPC) tissues [5]. Overall, these PDXs recapitulate the heterogeneity of the patients' original tumors in a high-fidelity manner [5]. More excitingly, the HNPC PDXs are also able to closely mimic the original tumor's biological behaviors and disease progression, including development into CRPC following mouse host castration. Thus, such HNPC-to-CRPC PDXs provide an excellent opportunity to model CRPC progression and analyze gene expression changes longitudinally during this process (i.e., at multiple time points precastration, post-castration, and when fully-relapsed). This makes the identification of potential early driver genes that critically contribute to CRPC development possible.

In this study, we mimic CRPC progression using seven HNPC PDX models (i.e., LTL310, 311, 313B, 313H, 418, 467, and 484). Following host mouse castration, these HNPC PDXs spontaneously develop into their CRPC forms. PDX tissues were collected at various time points during this developmental process and subjected to comparative transcriptomic analysis. We found that ZRSR2 is consistently and significantly upregulated in our PDX models during CRPC development and is also elevated in multiple clinical CRPC cohorts. ZRSR2 is short for zinc finger CCCH-type, RNA binding motif and serine/arginine rich 2 and encodes a splice factor involved in the recognition of 3 '-intron splice sites. ZRSR2 has not been previously reported as involved in solid cancers but sees frequent mutations in hematologic malignancies [6]. Here, we provide evidence that ZRSR2 could be an early driver of CRPC development.

\section{Materials and methods}

\section{PDX models and microarray data}

This study followed the ethical guidelines stated in the Declaration of Helsinki. Specimens were obtained from patients with their informed written consent following a protocol (\#H09-01628) approved by the Institutional Review Board at the University of British Columbia (UBC).
Animal studies were approved by UBC's Animal Care and Use Committee under protocol \#A17-0165. All PCa PDXs used in this study were previously established in male nonobese diabetic/severe combined immunodeficient (NOD/ SCID) mice (NOD.CB17-Prkdcscid/J) and stored in liquid nitrogen as frozen seeds. Gene expression analysis was performed by microarray using the GE $8 \times 60 \mathrm{~K}$ Microarray as previously described [5].

Primers for qRT-PCR used in this study are listed in Supplementary Table 1. Detailed information for other materials and methods is provided in Supplementary Information.

\section{Results}

\section{ZRSR2 overexpression is an early and consistent event during CRPC development in PDXs}

We have previously developed a series of PDX models derived from patient HNPC tissues. These PDXs show high fidelity to the original patient tumors with respect to morphologies, genomics, gene expression levels, and treatment responses. They also mimic the clinical process of CRPC development [5]. For example, in the LTL-313H model, host castration resulted in a marked inhibition of AR signaling as indicated by a decrease in serum PSA levels and PSA protein expression. The LTL-313HR CRPC PDX develops $\sim 5$ months after host castration (Fig. 1a, b). Since tumor samples can be collected at different time points throughout this process, these PDX models allow us to carry out longitudinal analyses of CRPC development and offer a unique tool for tracing early molecular changes.

Samples of the different PDX models were collected at the initial HNPC stage, 12 weeks after castration $(\mathrm{Cx})$, and when CRPC fully develops. Microarray analysis was performed to explore potential gene candidates driving CRPC development. Our previous study found a set of 18 genes that are significantly and consistently upregulated in 10 paired Cx vs. HNPC and 7 paired CRPC vs. HNPC PDX models [7]. Here, we assessed the expression levels of these 18 genes in the Cambridge 2015 clinical cohort. ZRSR2 was the top-ranked gene that showed significant differential expression between clinical HNPC and CRPC based on $p$ values (Fig. 1c, d). Thus, this data demonstrates that ZRSR2 is a significantly upregulated gene before CRPC fully develops and its expression levels remain consistently elevated in CRPC tumors.

\section{Elevated ZRSR2 expression is observed in multiple CRPC PDX models and patient samples}

ZRSR2 mRNA expression is upregulated in multiple castrated PDX models when compared to the parental HNPC PDXs 
a

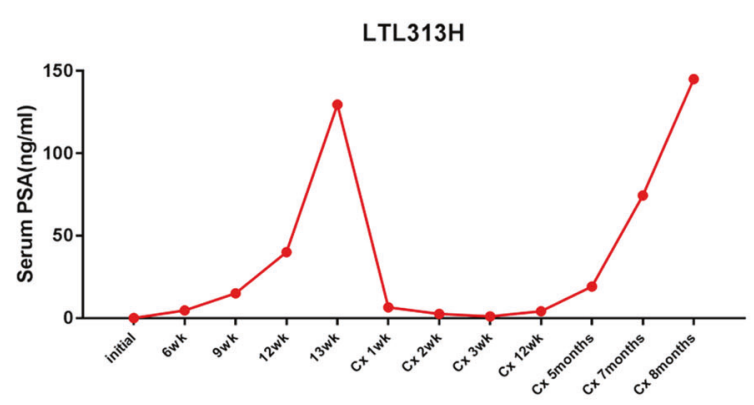

C

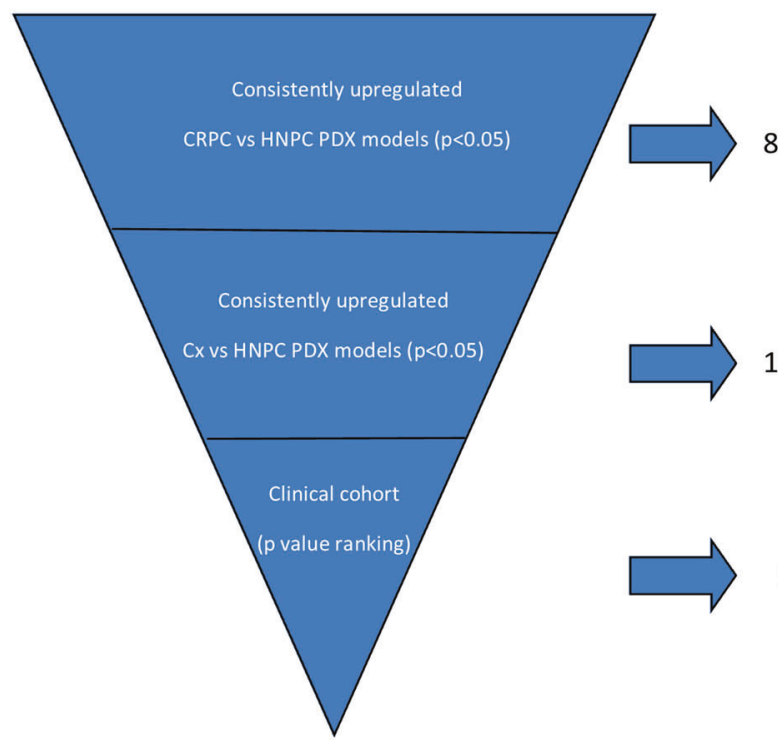

Fig. 1 Identification of $Z R S R 2$ overexpression as a potential early and consistent event during CRPC development. a Mouse serum PSA levels at various time points before, during, and after CRPC development in the LTL-313H model. b HE staining of LTL-313H tumor sections with immunohistochemistry showing the expression levels of Ki67, AR, and PSA in tumor sections at various time points. c, d The strategy used to identify ZRSR2 overexpression as a potential

(Fig. 2a). Similarly, ZRSR2 protein expression is significantly increased during CRPC development in LTL-313H (Fig. 2b). By analyzing several publicly available clinical datasets [8], ZRSR2 is also found to be elevated in clinical CRPC compared to HNPC in multiple cohorts (Michigan 2005, Michigan 2012, and Cambridge) [9-11] (Fig. 2c-e). Meanwhile, patients exhibiting elevated ZRSR2 expression show shorter progression-free survival times [12] (Fig. 2f).

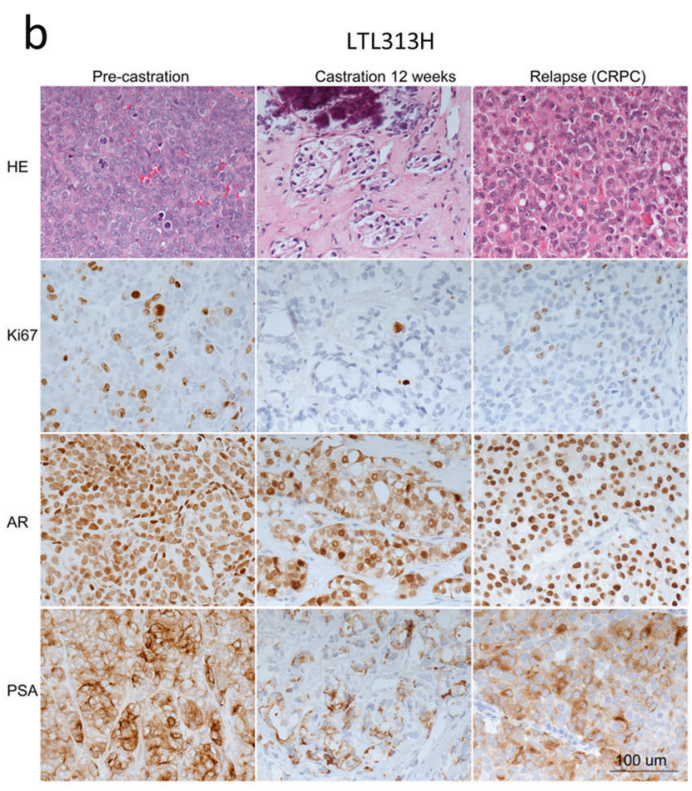

d

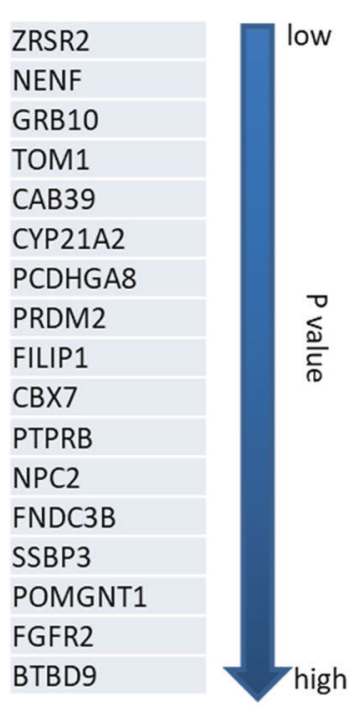

early and consistent event. Briefly, an 18-gene gene set was sorted for consistent upregulation in our CRPC PDX models compared to HNPC and at 12 weeks post-castration. Then, the $p$ values of these genes were determined in a clinical cohort comparing CRPC and HNPC samples. ZRSR2 was identified as the most significantly upregulated gene (lowest $p$ value) in the CRPC clinical cohort.

\section{Androgen deprivation therapy induces ZRSR2 expression in PCa cells}

We first determined the level of ZRSR2 expression in multiple PCa cell lines, including the lineage-related AR + LNCaP, C4-2, and MR49F cells. We found that ZRSR2 expression is low in the androgen-dependent $\mathrm{LNCaP}$ cells, higher in androgen-independent C4-2 cells, and highest in 


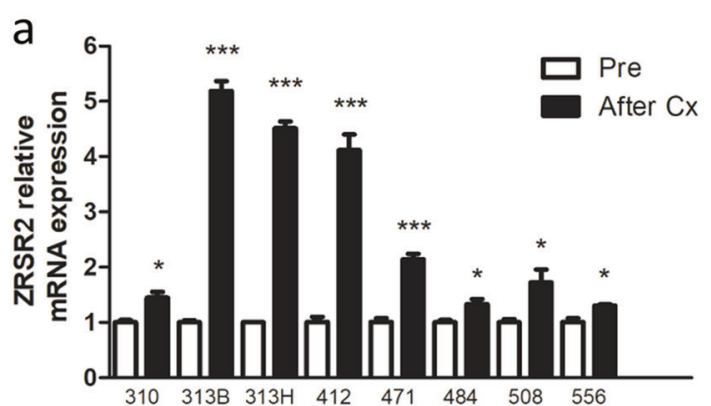

C

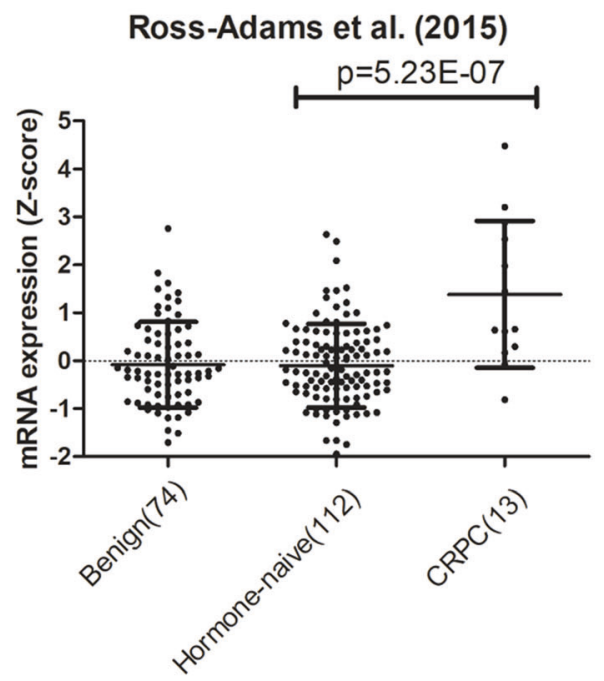

e

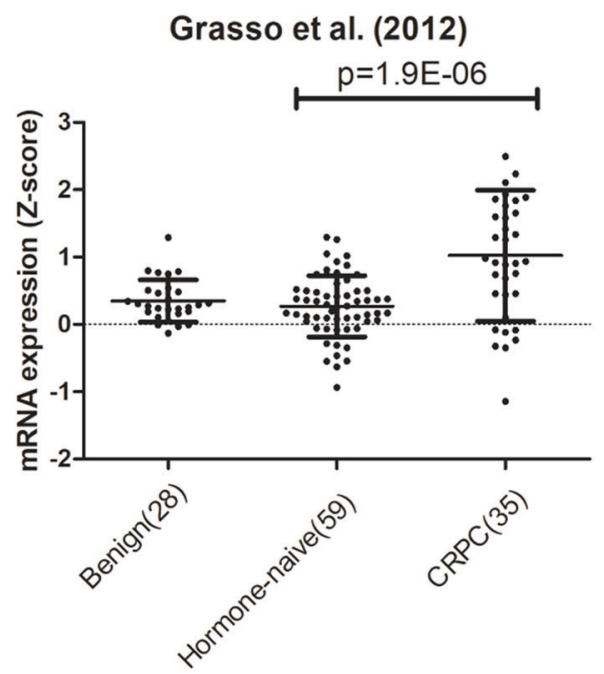

Fig. 2 Validation of ZRSR2 overexpression in PDX and patient CRPC tissues. a qRT-PCR was used to determine the expression of ZRSR2 in multiple PDX lines after host castration. The results are presented as means \pm SEM. $p$ values for each sample were determined based on paired parental tumors $(* p<0.05$; $* * p<0.01$; $* * * p<0.001)$. b ZRSR2 protein expressions in LTL-313H tumor tissues at HNPC, 12 weeks after castration, and after CRPC development were b

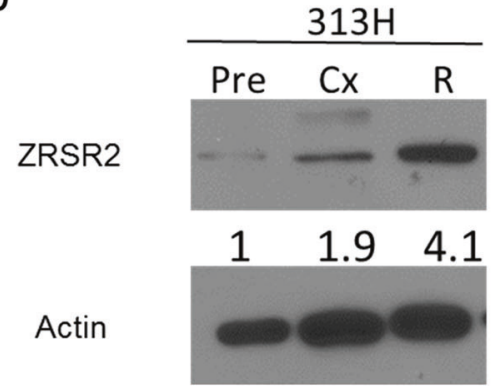

d

Varambally et al. (2005)

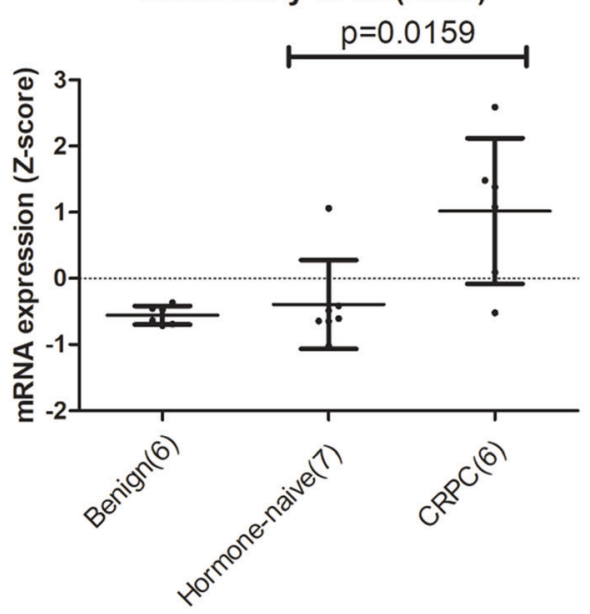

$f$

$\operatorname{TCGA}(2015)$

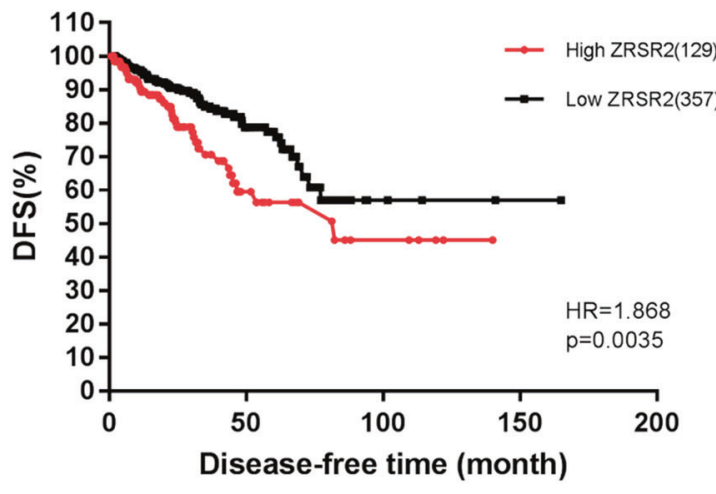

determined by western blotting. c, d, e ZRSR 2 mRNA expressions in benign prostate tissue, HNPC tissue, and CRPC tissue from three independent clinical cohorts. The vertical scatter plots show means \pm SD. f Kaplan-Meier plots indicating disease-free survival times of PCa patients grouped according to ZRSR2 expression. Significance between the groups was analyzed by the log-rank test. 


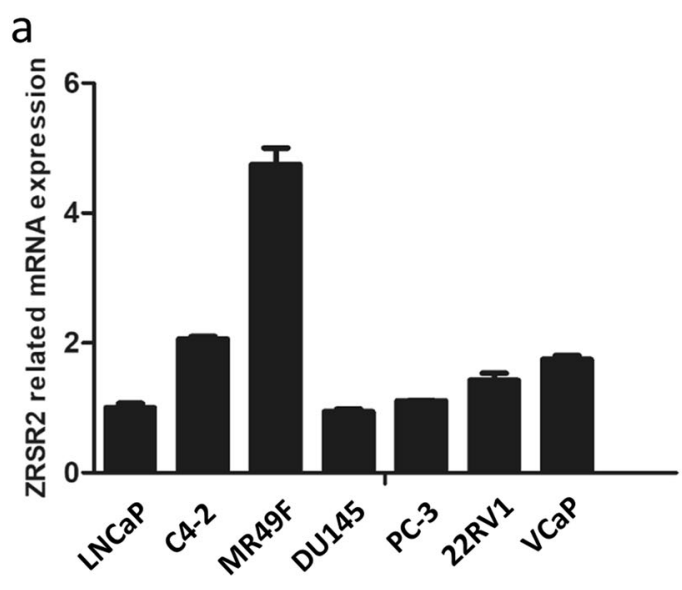

b

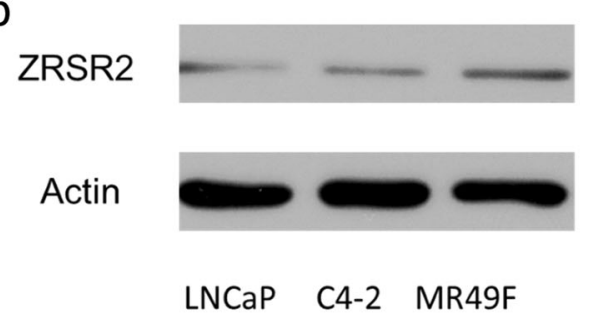

C
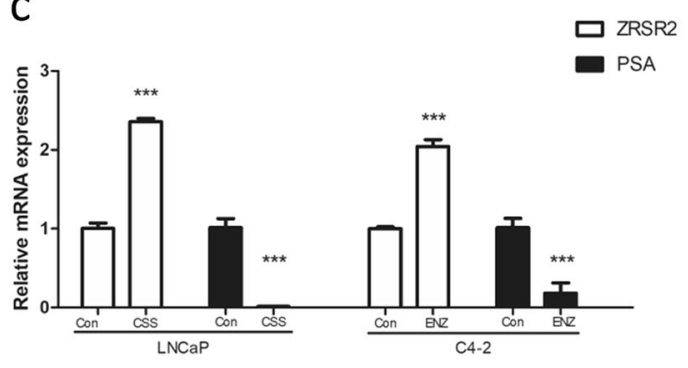

d

$\frac{\text { LNCaP }}{\text { FBS CSS }}$
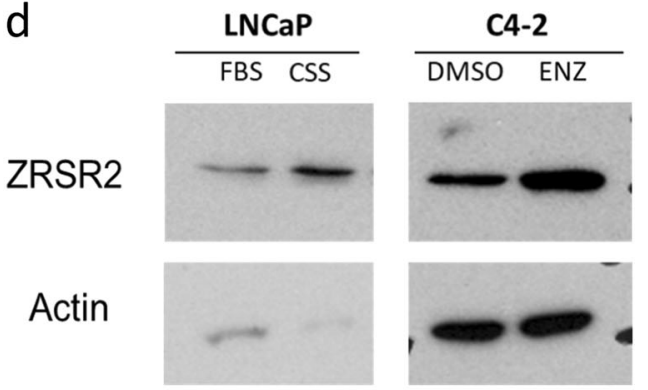

e

Shaw et al (2016)

f
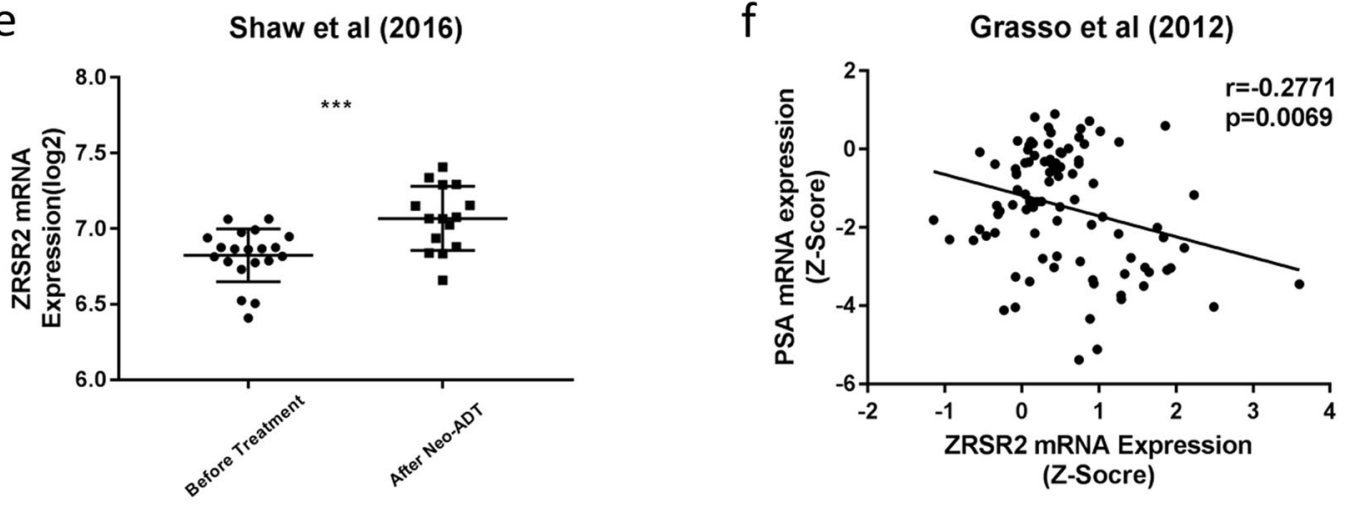

g

Ross-Adams et al (2015)

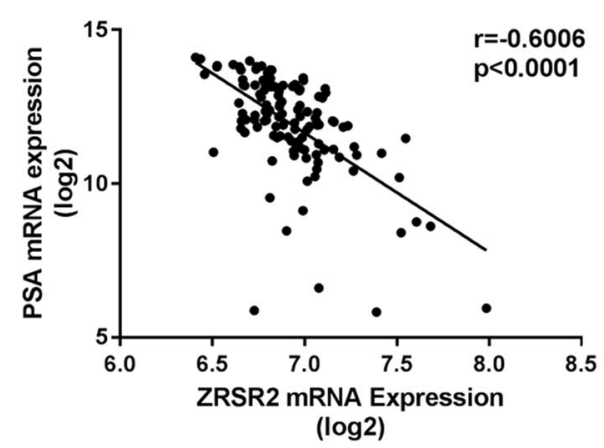

the ENZ-resistant MR49F cells at both the mRNA and protein levels. This suggests that $Z R S R 2$ expression in these cell lines is inversely associated with androgen

independence (Fig. 3a, b). In order to examine whether ZRSR2 expression is functionally related to androgen activity, we performed androgen ablation in LNCaP cells 
Fig. 3 Androgen deprivation therapy induces $Z R S R 2$ expression in PCa cells. a ZRSR2 mRNA expressions in multiple PCa cell lines were determined by qRT-PCR. The results are presented as means \pm SEM. b ZRSR2 protein expressions in the human lineage-related AR + PCa LNCaP, C4-2 and MR49F cells were determined by western blotting. c ZRSR 2 mRNA expressions in LNCaP cells after androgen deprivation treatment for 5 days (cultured in CSS medium) and in C42 cells after ENZ treatment for 5 days were determined by qRT-PCR. The results are presented as means \pm SEM. d ZRSR2 protein expressions in LNCaP cells after androgen deprivation therapy for 5 days (cultured in CSS medium) and in C4-2 cells after ENZ treatment for 5 days were determined by western blotting. e The early effect of neoadjuvant ADT (Neo-ADT) on ZRSR2 mRNA expression in clinical $\mathrm{PCa}$. The vertical scatter plots show means \pm SD. f, $\mathbf{g}$ Pearson correlation between $Z R S R 2$ and $K L K 3$ (PSA) mRNA expressions was determined using data from public clinical cohorts. The trend line is shown.

and androgen receptor inhibition in C4-2 cells. In androgensensitive LNCaP cells, ZRSR2 mRNA and protein expressions were elevated after androgen ablation. In androgenindependent but AR signaling-active C4-2 cells, ENZ treatment also led to an upregulation of ZRSR2 expression (Fig. 3c, d). Additionally, we found that ZRSR2 expression is significantly upregulated in patient samples collected after neoadjuvant ADT when compared to HNPC samples [13] (Fig. 3e). Furthermore, the expression of ZRSR2 is significantly negatively correlated with PSA expression in two independent clinical cohorts [10, 11] (Fig. 3f, g).

\section{Knockdown of ZRSR2 inhibits the proliferation of PCa cells}

Following the observation that $Z R S R 2$ is upregulated in CRPC, we subsequently utilized LNCaP, C4-2, and ENZresistant 22Rv1 cells to determine its functional role. Two siRNAs were used to knockdown ZRSR2. The qPCR and western blot results indicate that both sequences can successfully knockdown ZRSR2 expression in these cells (Fig. 4a). MTS proliferation assay showed that ZRSR2 silencing inhibited cell proliferation (Fig. 4b). Similar results were also observed using the crystal violet staining assay (Fig. 4c). ZRSR2 knockdown inhibited cell proliferation more dramatically in androgen-independent C4-2 and 22Rv1 cells than in androgen-sensitive LNCaP cells.

\section{Knockdown of ZRSR2 inhibits DNA synthesis and delays cell cycle progression in PCa cells}

Knockdown of ZRSR2 decreased DNA synthesis as indicated by a reduction in EdU-positive cells, suggesting a delayed cell cycle progression (Fig. 4d, e). Moreover, PI staining indicated that there is an increase of cells in the G0/ G1 phase and a decrease of cells in the $S$ phase following ZRSR2 knockdown (Fig. 4f).
ZRSR2 knockdown decreases CCND1 expression in PCa cells

Our data show that ZRSR2 can affect G1 to S phase progression in the cell cycle. D-type cyclins are regulators of G1-S progression in mammalian cells [14]. Knockdown of ZRSR2's partner SF3b1 can decrease the levels of Cyclin D1 (CCND1) by binding U2 small nuclear ribonucleoproteins (U2 snRNP) to Tat-SF1 [15]. CCND1 is a well-known proto-oncogene in many cancers. The CCND1-related signaling pathway is also significantly upregulated in CRPC [16]. Based on studies that investigated ZRSR2 structure and binding functions, we hypothesize that ZRSR2's effect on cell cycle progression in PCa may be mediated by CCND1. Here, we observed that knockdown of ZRSR2 in LNCaP, C4-2, and 22Rv1 cells consistently led to a decrease in CCNDl mRNA and protein expression (Fig. 5a, b). Furthermore, in clinical PCa samples, ZRSR2 expression is positively correlated with CCNDI expression in both the Cambridge and Grasso clinical cohorts (Fig. 5c). As a splicing factor, ZRSR2 knockdown did not show affect ARV7 expression (Fig. 5d). This data suggest that upregulated expression of ZRSR2 in CRPC might enhance CCND1 expression, which in turn promotes cell proliferation.

\section{Discussion}

The development of CRPC is currently a major hurdle in the management of advanced PCa [4]. A better understanding of the molecular mechanisms underlying the development of CRPC is thus needed. However, the lack of clinically relevant models, especially models based on patient-derived HNPC, is a challenge to PCa research. This is partially due to the low success rate of $\mathrm{PCa}$ tissue xenograft model development. Most engraftments only succeed when advanced cancers with high growth rates are used [17, 18]. To better understand the progression of CRPC, our laboratory has established 49 transplantable PCa PDX models, including models of HNPC, neuroendocrine (NE) $\mathrm{PCa}$, and CRPC. We achieved this by directly grafting the patient's tumor under the mouse renal capsule. Importantly, these PDX models show high fidelity to the original patient tumor in morphology, genomics, gene expression, and treatment response. In particular, they can mimic the progression of HNPC to CRPC upon host castration. With these PDX models, we were able to identify the common molecular events that occur during CRPC development $[5,7]$. Understanding early events in tumor development can better guide disease management and control. However, these early events are difficult to identify due to the long latency in tumor development and the various limitations of PCa modeling. Our PDXs offer an excellent platform to 

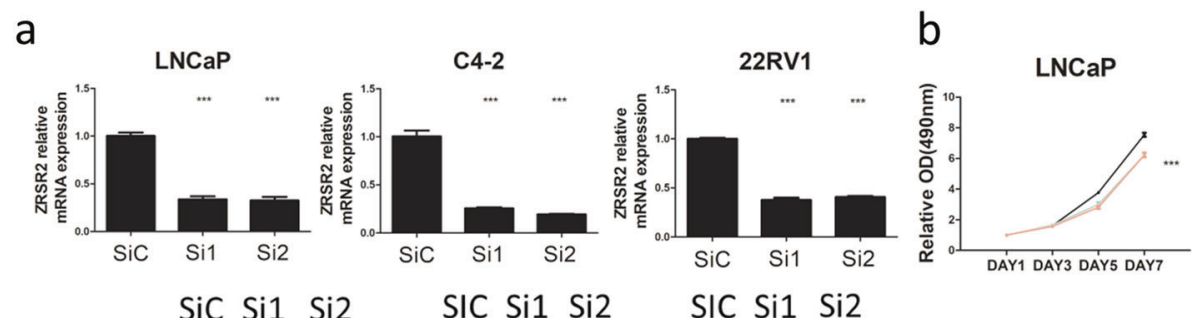

SiC Si1 Si2
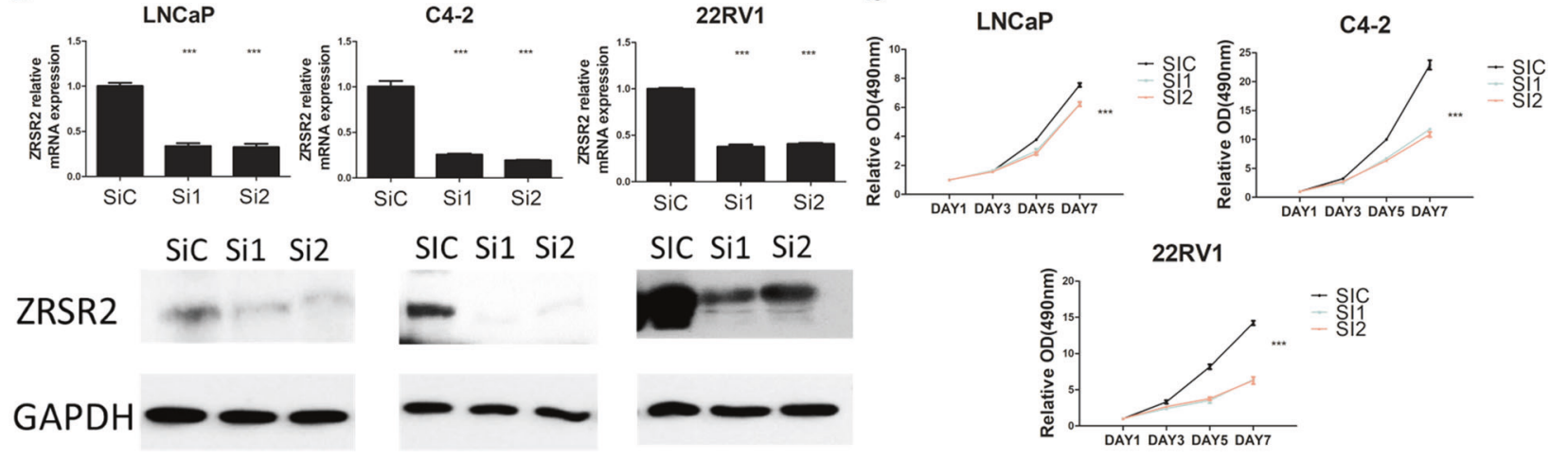

C
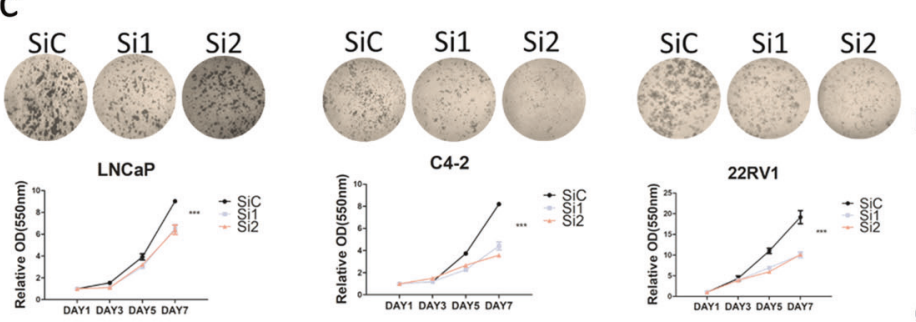

e
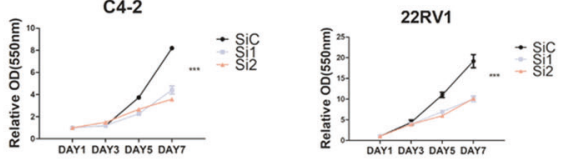

LNCaP
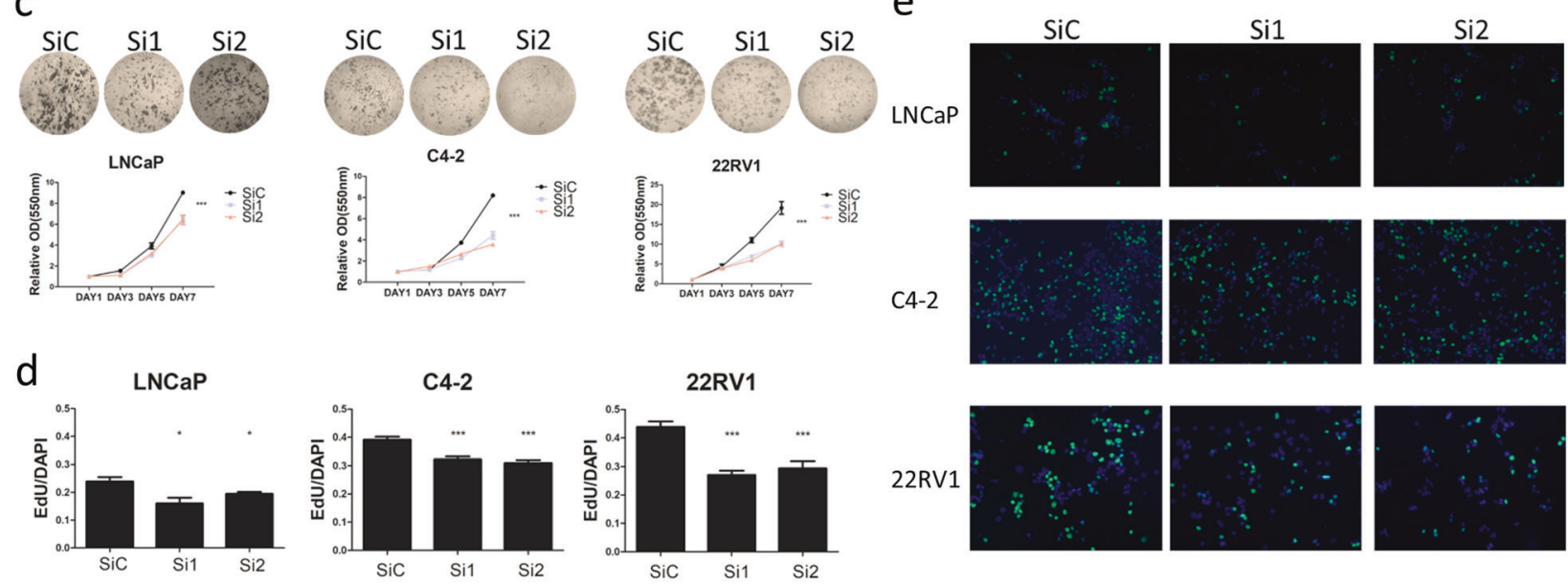

C4-2
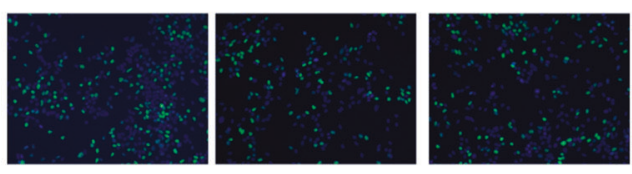

f
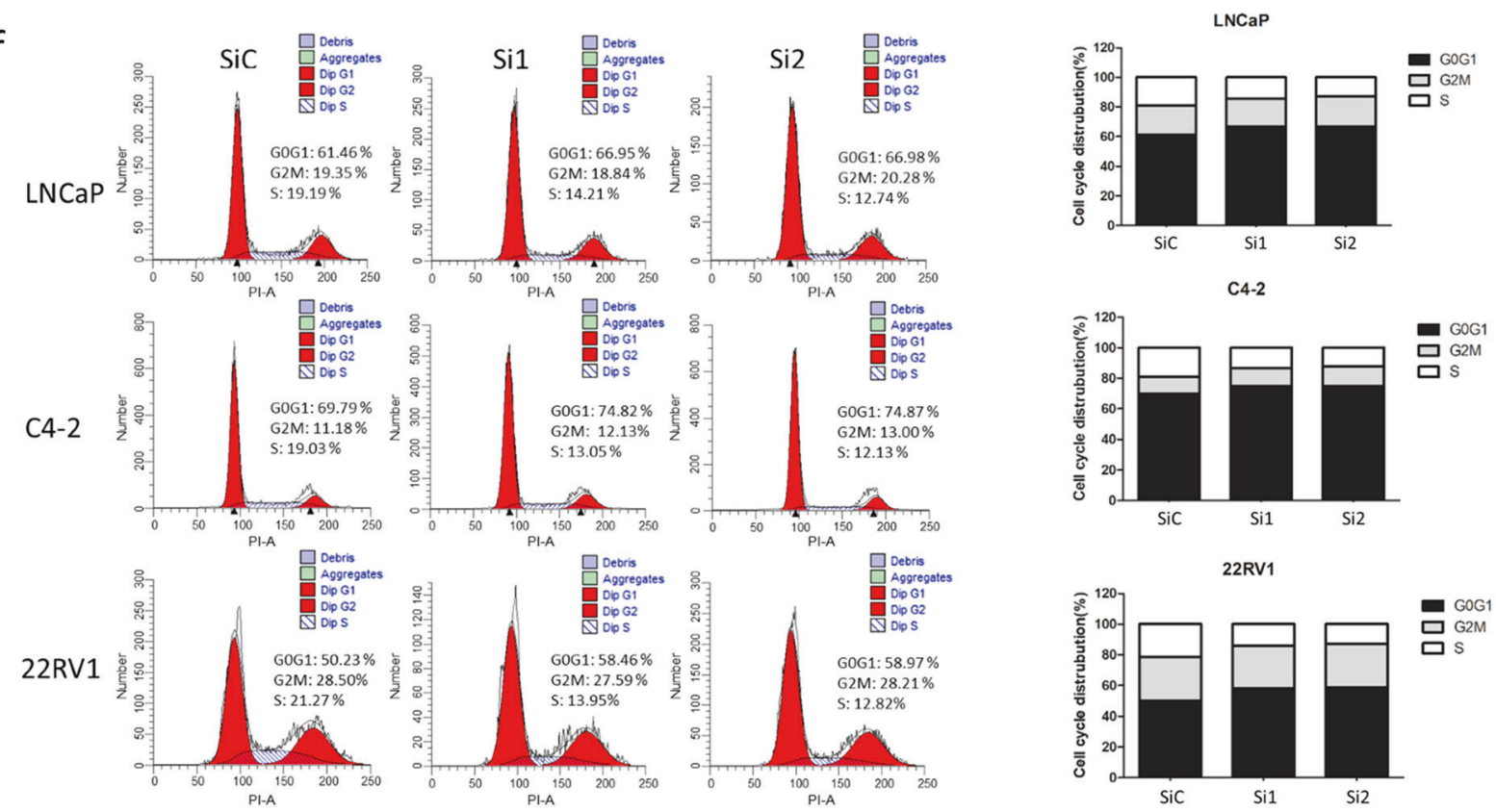

overcome such issues. Tumor tissues from different time points following treatment can be collected for further study. Using our PDX models, we were able to identify early drivers that promoted $\mathrm{NE}$ transdifferentiation in prostate adenocarcinoma [19]. In this paper, using paired PDX models combined with clinical cohorts, we discovered ZRSR2 as a potential early driver gene in CRPC development. 
Fig. 4 ZRSR2 knockdown inhibits the proliferation, DNA synthesis and delays cell cycle progression of PCa cells. a The ability of two siRNAs to knockdown ZRSR2 was confirmed by qRT-PCR and western blotting. The qRT-PCR results are presented as means \pm SEM. b The proliferative effects of ZRSR2 expression in LNCaP, C4-2, and 22Rv1 cells were determined by the MTS cell proliferation assay. OD values at $490 \mathrm{~nm}$ were normalized based on Day 1 readings. Results are presented as means \pm SEM. c The proliferative effects of $Z R S R 2$ expression in LNCaP, C4-2, and 22Rv1 cells were further determined by the crystal violet staining assay. OD values at $550 \mathrm{~nm}$ were normalized based on Day 1 readings. Results are presented as means \pm SEM. d, e The effects of ZRSR2 knockdown on DNA synthesis were determined by the EdU assay. EdU-labeled cells (green) and total cells (counterstained with DAPI; blue) were counted with 10 images taken at 10 -fold magnification. Bars (EdU positive/total cells) show means \pm SEM. f FACS analysis of LNCaP, C4-2, and 22Rv1 cells after ZRSR2 knockdown. Quantitation of cell cycle is shown in the bar graph.

It is well-accepted that AR plays a major role in the progression of a majority of CRPCs [4]. In this study, we identified that ZRSR2 expression is induced during ADT or ARPI treatment and remains elevated in AR + CRPCs. In addition, our study shows that ZRSR2 is critical for $\mathrm{AR}+\mathrm{PCa}$ cell proliferation. As shown in Fig. 4b, c, knocking down ZRSR2 in C4-2 and 22Rv1 CRPC cells is more effective at reducing cell proliferation than in LNCaP cells. This suggests that ZRSR2 may be essential to CRPC cells. To check the AR activity after ZRSR2 knockdown, we examined the AR target gene expression. However, there is no significant alterations with the AR target gene KLK2, KLK3, TMPRSS2 and FKBP5 after ZRSR2 knockdown (Supplementary Fig. 1). It suggested that ZRSR2-induced proliferation can be independent of AR/AR activity in CRPC.

ZRSR2 is frequently mutated in myeloid malignancies and in $\sim 5 \%$ of patients with myelodysplastic syndromes (MDS). However, ZRSR2 mutations have also been found at a lower frequency in a variety of non-solid tumors [20, 21]. This study reports, for the first time, a potentially important functional role for $Z R S R 2$ in solid tumors. Using our unique panel of HNPC PDX models, ZRSR2 was not only found to be upregulated in fully developed CRPC PDXs, its upregulation is also an early event after host castration during CRPC development. ZRSR2 is rarely mutated in PCa according to public datasets in cBioPortal. Madan et al. reported that the downregulation of ZRSR2 impaired in vitro clonogenic ability and suppressed tumor formation in mice. ZRSR2 knockdown in erythroleukemia TF-1 and K562 cells showed a general reduction in cell growth, with fewer cells detected in the $\mathrm{S}$ phase of the cell cycle [6]. ZRSR2 knockdown in HeLa cells also reduced cell viability [22]. Similarly, in our study, knockdown of ZRSR 2 in PCa cell lines inhibited cell proliferation, with an increase of cells in the G0/ G1 phase and a decrease of cells in S phase.

ZRSR2 is an essential splicing factor involved in the recognition of 3 '-intron splice sites [22]. One known mechanism of ARPI-resistance is alternative AR splicing, with AR-V7 being the most common alternative splicing product [23]. Although ZRSR2 knockdown effectively reduced cell growth in the AR-V7 highly-expressed 22Rv1 cells, AR-V7 expression was itself not affected (Fig. 5d). ZRSR2 is a serine/arginine-rich (SR-rich) splicing factor and interacts with other SR proteins to perform its essential functions in RNA splicing [6]. Although there are no reports concerning ZRSR2 functions in solid tumors, an increasing number of studies have reported that SR proteins and their specific kinases (SRPKs) as partners to ZRSR2mediated splicing can have oncogenic effects in several malignancies such as lung cancer, breast cancer and $\mathrm{PCa}$ [24-27]. There are currently no known mechanisms regarding how ZRSR2 mediates CRPC development. Based on our study, ZRSR2 can facilitate cell proliferation in $\mathrm{PCa}$ cell lines and promote G1-to-S phase progression. Some studies reported that ZRSR2's partner SRSF2 can affect $C C N D 1$ splicing in $\mathrm{PCa}$, while another partner SRPK2 can trigger cell cycle progression in neurons and induce apoptosis through regulation of nuclear CCND1 [27, 28]. As mentioned in the results section, knockdown of SF3b1 can decrease the levels of CCND1 by binding U2 snRNP to TatSF1 [15]. ZRSR2 also interacts with SF3B1 bound to U2 snRNP [21]. A recent report demonstrated that the CCND1 signaling pathway is significantly upregulated in drug-resistant PCa cells [16]. Here, we hypothesize that ZRSR2 may affect cell proliferation and cell cycle progression by mediating $C C N D 1$ expression during CRPC development. This is supported by our findings of a positive correlation between ZRSR2 and CCNDI expression in clinical cohorts, and a decreased expression of $C C N D 1$ induced by ZRSR2 knockdown.

In summary, ZRSR2 showed early and consistently overexpressed during CRPC development. ADT/ARPI treatment could induce ZRSR2 expression. As we observed, ZRSR2 was mainly associated with PCa proliferation. We hypothesized that ZRSR2 might be a promoter that is upregulated when ADT/ARPI treatment is assigned. It could protect cell proliferation and antagonize the effect of drugs then promote CRPC development. As we mentioned, most of the antiCRPC therapies were focusing on late stage of the disease. Since the increased ZRSR2 expression was observed in early stage of CRPC development in our PDXs and clinical cohorts. It suggests an opportunity to develop a therapeutic agent that could delay or halt the development of CRPC by targeting ZRSR2 gene in combination of ADT treatment.

\section{Conclusion}

Using PDX models, we identified, for the first time, that ZRSR2 overexpression is an early event in CRPC 


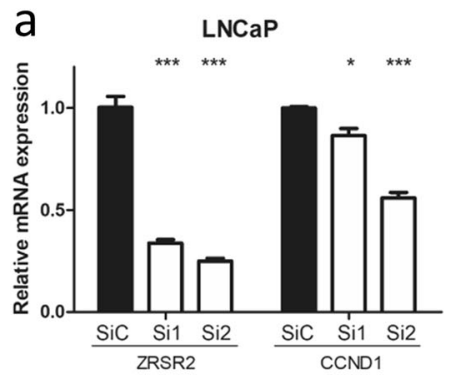

b

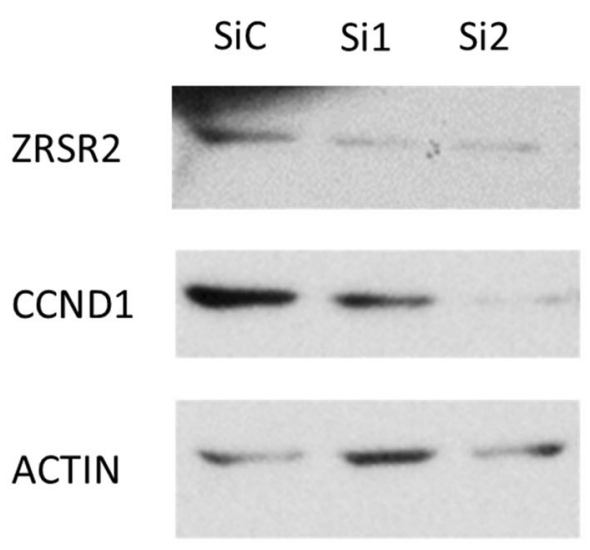

C

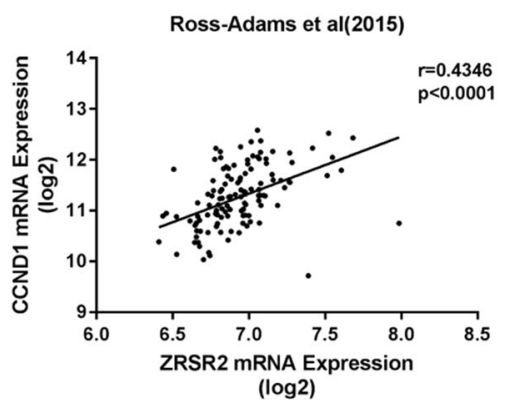

d

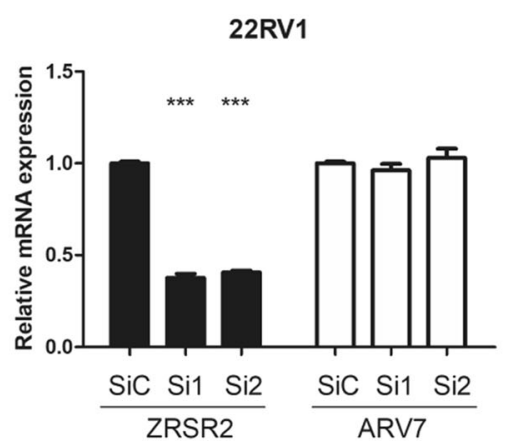

C4-2

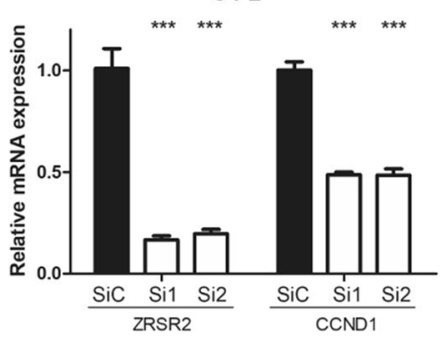

C4-2

Sic Si1 Si2
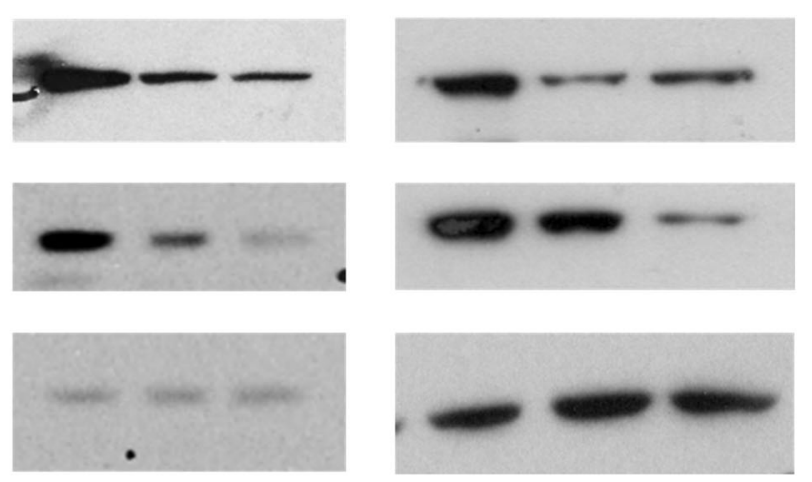

Grasso et al(2012)

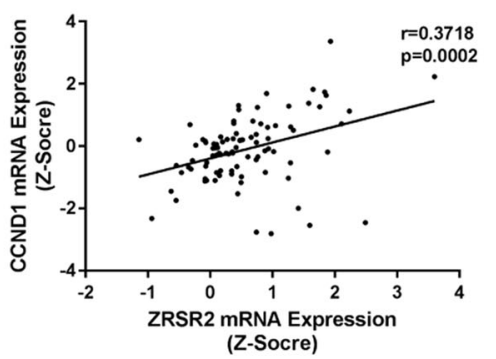

Fig. 5 ZRSR2 knockdown affects CCND1 expression in PCa cells. a The mRNA expressions of CCND1 after ZRSR2 knockdown in LNCaP, C4-2, and 22Rv1 cells were determined by qRT-PCR. The results are presented as means \pm SEM. b The effects of $C C N D 1$ protein expression after ZRSR2 knockdown in LNCaP, C4-2, and 22Rv1 cells were determined by western blotting. c Pearson correlation between ZRSR2 and CCND1 mRNA expressions was determined using data from public clinical cohorts. The trend line is shown. $\mathbf{d}$ The effects of AR-V7 mRNA expression after ZRSR2 knockdown in the 22Rv1 cells were determined by qRT-PCR. The results are presented as means \pm SEM. 
development. Elevated ZRSR2 expression is associated with poor outcomes in PCa patients. It plays an important role in CRPC development and might be implicated in CCND1 signaling in $\mathrm{PCa}$.

Acknowledgements We would like to thank all members of the Living Tumor Laboratory for technical support and helpful discussions.

Author contributions Study concept and design: HH, JH, DL, and YZW. Acquisition of data: HH, JH, XD, YW, RW, and CC. Analysis and interpretation of data: $\mathrm{HH}, \mathrm{JH}, \mathrm{XC}, \mathrm{MS}$, DL, and CC. Drafting of the manuscript: $\mathrm{HH}, \mathrm{JH}$, and SYCC. Critical revision of the manuscript for important intellectual content: $\mathrm{HH}, \mathrm{JH}, \mathrm{SQ}$, DL, and YZW. Statistical analysis: $\mathrm{HH}, \mathrm{JH}$, and $\mathrm{YW}$. Obtaining funding: $\mathrm{CC}$ and YZW. Administrative, technical, or material support: XD, HX, RW. Supervision: XZ, DL, and YZW.

Funding This research was supported in part by the Canadian Institutes of Health Research (\#141635, \#144159, \#153081, \#173338, YZW), Terry Fox Research Institute (\#1062, YZW), Mitacs Accelerate Program (\#IT10125, \#IT06414, \#IT12387, \#IT14958, YZW), NCI grant (P50CA097186) pilot project award (YZW). HH is sponsored by the China Scholarship Council as a joint training Ph.D. student at the University of British Columbia (ID: 201706370229). The funder (China Scholarship Council) had no role in the study design, data collection, and analysis, decision to publish, or preparation of the manuscript.

\section{Compliance with ethical standards}

Conflict of interest The authors declare that they have no conflict of interest.

Publisher's note Springer Nature remains neutral with regard to jurisdictional claims in published maps and institutional affiliations.

Open Access This article is licensed under a Creative Commons Attribution 4.0 International License, which permits use, sharing, adaptation, distribution and reproduction in any medium or format, as long as you give appropriate credit to the original author(s) and the source, provide a link to the Creative Commons license, and indicate if changes were made. The images or other third party material in this article are included in the article's Creative Commons license, unless indicated otherwise in a credit line to the material. If material is not included in the article's Creative Commons license and your intended use is not permitted by statutory regulation or exceeds the permitted use, you will need to obtain permission directly from the copyright holder. To view a copy of this license, visit http://creativecommons. org/licenses/by/4.0/.

\section{References}

1. Chatterjee B. The role of the androgen receptor in the development of prostatic hyperplasia and prostate cancer. Mol Cell Biochem. 2003;253:89-101.

2. Smith MR, Saad F, Chowdhury S, Oudard S, Hadaschik BA, Graff JN et al. Apalutamide treatment and metastasis-free survival in prostate cancer. N Engl J Med. 2018;378:1408-18.

3. Liu JM, Yu CP, Chuang HC, Wu CT, Hsu RJ. Androgen deprivation therapy for prostate cancer and the risk of autoimmune diseases. Prostate Cancer Prostatic Dis. 2019; 22:475-82.
4. Yuan X, Cai C, Chen S, Chen S, Yu Z, Balk SP. Androgen receptor functions in castration-resistant prostate cancer and mechanisms of resistance to new agents targeting the androgen axis. Oncogene. 2014;33:2815-25.

5. Lin D, Wyatt AW, Xue H, Wang Y, Dong X, Haegert A, et al. High fidelity patient-derived xenografts for accelerating prostate cancer discovery and drug development. Cancer Res. 2014;74:1272-83.

6. Madan V, Kanojia D, Li J, Okamoto R, Sato-Otsubo A, Kohlmann A, et al. Aberrant splicing of U12-type introns is the hallmark of ZRSR2 mutant myelodysplastic syndrome. Nat Commun. 2015;6:6042.

7. Hao J, Ci X, Xue H, Wu R, Dong X, Choi SYC, et al. Patientderived hormone-naive prostate cancer xenograft models reveal growth factor receptor bound protein 10 as an androgen receptorrepressed gene driving the development of castration-resistant prostate cancer. Eur Urol. 2018;73:949-60.

8. Pavía-Jiménez A, Tcheuyap VT, Brugarolas J. Establishing a human renal cell carcinoma tumorgraft platform for preclinical drug testing. Nat Protoc. 2014;9:1848-59.

9. Varambally S, Yu J, Laxman B, Rhodes DR, Mehra R, Tomlins $\mathrm{SA}$, et al. Integrative genomic and proteomic analysis of prostate cancer reveals signatures of metastatic progression. Cancer Cell. 2005;8:393-406.

10. Grasso CS, Wu YM, Robinson DR, Cao X, Dhanasekaran SM, Khan AP, et al. The mutational landscape of lethal castrationresistant prostate cancer. Nature. 2012;487:239-43.

11. Ross-Adams H, Lamb AD, Dunning MJ, Halim S, Lindberg J, Massie CM, et al. Integration of copy number and transcriptomics provides risk stratification in prostate cancer: a discovery and validation cohort study. EBioMedicine. 2015;2:1133-44.

12. Cerami E, Gao J, Dogrusoz U, Gross BE, Sumer SO, Aksoy BA, et al. The cBio cancer genomics portal: an open platform for exploring multidimensional cancer genomics data. Cancer Discov. 2012;2:401-4.

13. Shaw GL, Whitaker H, Corcoran M, Dunning MJ, Luxton H, Kay $\mathrm{J}$, et al. The early effects of rapid androgen deprivation on human prostate cancer. Eur Urol. 2016;70:214-8.

14. Diehl JA. Cycling to cancer with cyclin D1. Cancer Biol Ther. 2002;1:226-31.

15. Loerch S, Leach JR, Horner SW, Maji D, Jenkins JL, Pulvino MJ et al. The pre-mRNA splicing and transcription factor Tat-SF1 is a functional partner of the spliceosome SF3b1 subunit via a U2AF homology motif interface. J Biol Chem. 2019;294:2892-902.

16. Pal SK, Patel J, He M, Foulk B, Kraft K, Smirnov DA, et al. Identification of mechanisms of resistance to treatment with abiraterone acetate or enzalutamide in patients with castrationresistant prostate cancer (CRPC). Cancer. 2018;124:1216-24.

17. van Weerden WM, de Ridder CM, Verdaasdonk CL, Romijn JC, van der Kwast TH, Schröder FH, et al. Development of seven new human prostate tumor xenograft models and their histopathological characterization. Am J Pathol. 1996;149:1055-62.

18. Klein KA, Reiter RE, Redula J, Moradi H, Zhu XL, Brothman $\mathrm{AR}$, et al. Progression of metastatic human prostate cancer to androgen independence in immunodeficient SCID mice. Nat Med. 1997;3:402-8.

19. Ci X, Hao J, Dong X, Choi SY, Xue H, Wu R, et al. Heterochromatin protein $1 \alpha$ mediates development and aggressiveness of neuroendocrine prostate cancer. Cancer Res. 2018;78:2691-704.

20. Bejar R. Splicing factor mutations in cancer. Adv Exp Med Biol. 2016;907:215-28.

21. Yoshida K, Sanada M, Shiraishi Y, Nowak D, Nagata Y, Yamamoto R, et al. Frequent pathway mutations of splicing machinery in myelodysplasia. Nature. 2011;478:64-69.

22. Shen H, Zheng X, Luecke S, Green MR. The U2AF35-related protein Urp contacts the 3' splice site to promote U12-type intron 
splicing and the second step of U2-type intron splicing. Genes Dev. 2010;24:2389-94.

23. Chandrasekar T, Yang JC, Gao AC, Evans CP. Mechanisms of resistance in castration-resistant prostate cancer (CRPC). Transl Androl Urol. 2015;4:365-80.

24. Zerbe LK, Pino I, Pio R, Cosper PF, Dwyer-Nield LD, Meyer AM, et al. Relative amounts of antagonistic splicing factors, hnRNP A1 and ASF/SF2, change during neoplastic lung growth: implications for pre-mRNA processing. Mol Carcinog. 2004;41:187-96.

25. de Miguel FJ, Sharma RD, Pajares MJ, Montuenga LM, Rubio A, Pio R. Identification of alternative splicing events regulated by the oncogenic factor SRSF1 in lung cancer. Cancer Res. 2014;74: $1105-15$.

26. Anczuków O, Akerman M, Cléry A, Wu J, Shen C, Shirole NH, et al. SRSF1-regulated alternative splicing in breast cancer. Mol Cell. 2015;60:105-17.

27. Olshavsky NA, Comstock CE, Schiewer MJ, Augello MA, Hyslop T, Sette C, et al. Identification of ASF/SF2 as a critical, allele-specific effector of the cyclin D1b oncogene. Cancer Res. 2010;70:3975-84.

28. Jang SW, Liu X, Fu H, Rees H, Yepes M, Levey A, et al. Interaction of Akt-phosphorylated SRPK2 with 14-3-3 mediates cell cycle and cell death in neurons. J Biol Chem. 2009;284:24512-25. 